\title{
Type II Sensitivity Analysis in Solid Assignment Problems
}

\author{
K. Kavitha ${ }^{1} \&$ P. Pandian ${ }^{1}$ \\ ${ }^{1}$ Department of Mathematics, School of Advanced Sciences, VIT University, India \\ Correspondence: P. Pandian, Department of Mathematics, School of Advanced Sciences, VIT University, Vellore \\ 632014, India. E-mail: pandian61@rediffmail.com
}

Received: October 10, 2012

doi:10.5539/mas.v6n12p22
Accepted: November 2, $2012 \quad$ Online Published: November 14, 2012

URL: http://dx.doi.org/10.5539/mas.v6n12p22

\begin{abstract}
Type II sensitivity of the solid assignment problem is discussed in this paper. Parametric-bound method is proposed that determines the Type II sensitivity ranges of cost coefficients in the solid assignment problem. The procedure of the parametric-bound method is demonstrated with a numerical example. The result obtained by the proposed method will help the decision makers to take an appropriate action while handling various types of assignment problems having three parameters.
\end{abstract}

Keywords: solid assignment problem, Type II sensitivity analysis, parametric-bound method

\section{Introduction}

The assignment problem (AP) involving two parameters introduced by Votaw and Orden (1952) is a special type of a transportation problem and a linear zero-one programming problem. It is also one of the well-studied optimization problems which can be solved, using the linear programming technique, the transportation algorithm or the Hungarian method developed by Kuhn (1955). A solid assignment problem (SAP) consists of three parameters which is an extension of the AP. The solid assignment problems have wide applications in both manufacturing and service systems, multi-passive-sensor, capital investment, dynamic facility location, satellite launching and so on. In Pierskalla (1968), the SAP was proposed and solved using the branch and bound method. Frieze and Yadegar (1981) developed an algorithm for solving three-dimensional APs with application in scheduling. Recently, Anuradha and Pandian (2012) proposed a method for solving a SAP.

The sensitivity analysis (SA), one of the most interesting and preoccupying areas of optimization was studied by many researchers, using various algorithms. The assignment problem is a completely degenerate linear programming model. Chi-Jen Lin and Ue-Pyng Wen $(2003 ; 2007)$ studied sensitivity analysis of the assignment problem. The three types of sensitivity analysis of a fuzzy assignment problem, using labeling algorithm have been studied by Chi-Jen Lin et al. (2011).

This paper proposes a new method namely, parametric-bound method to find the Type II sensitivity range (SR) of SAP. Here, we show that the variables with positive optimal solutions are still positive and zero variables still unchanged in the SAP. The procedure of the parametric-bound method is demonstrated through a numerical example. The SA in a SAP by the proposed method can help the decision makers in evaluating the economical activities and making satisfactory decisions while handling a variety of three dimensional assignment problems.

\section{Solid Assignment Problem}

Consider $n$ jobs in $n$ factory and the factory has $n$ machines to process the jobs. Each job in a factory has to be associated with only one machine. A cost $c_{i j k}$ is incurred, when the job $j(j=1,2, \ldots, n)$ is processed by the machine $i \quad(i=1,2, \ldots, n)$ in the factory $k(k=1,2, \ldots, n)$. Let $x_{i j k}$ denote the assignment of jth job to ith machine in the kth factory. Our aim is to determine the assignment of jobs to machines at minimum assignment costs.

Now, the mathematical model of the above solid assignment problem (SAP) is given below:

Minimize

$$
z=\sum_{i=1}^{n} \sum_{j=1}^{n} \sum_{k=1}^{n} c_{i j k} x_{i j k}
$$

subject to 


$$
\begin{gathered}
\sum_{j=1}^{n} \sum_{k=1}^{n} x_{i j k}=1, i=1,2, \ldots, n \\
\sum_{i=1}^{n} \sum_{k=1}^{n} x_{i j k}=1, j=1,2, \ldots, n \\
\sum_{i=1}^{n} \sum_{j=1}^{n} x_{i j k}=1, k=1,2, \ldots, n \\
x_{i j k}=0 \text { (or) } 1, \text { for all } i, j \text { and } k
\end{gathered}
$$

where $c_{i j k}$ is the cost of assigning the job $\mathrm{j}$ to the machine $\mathrm{i}$ in the factory $\mathrm{k} . x_{i j k}=1$, if the job $\mathrm{j}$ is assigned to the machine $\mathrm{i}$ in the factory $\mathrm{k}$, and $x_{i j k}=0$, otherwise.

Any set of non-negative allocations to SAP which satisfies the Equations (1), (2), (3) and (4) is called a feasible solution to SAP. A feasible solution to SAP which minimizes the total assignment cost, that is, $\sum_{\mathrm{i}=1}^{\mathrm{n}} \sum_{\mathrm{j}=1}^{\mathrm{n}} \sum_{\mathrm{k}=1}^{\mathrm{n}} \mathrm{c}_{\mathrm{ijk}} \mathrm{x}_{\mathrm{ijk}}$ is called an optimal solution to the SAP.

\section{Sensitivity Analysis}

Sensitivity analysis is used to find out the effect of the changes in the value of the parameters and the structure of the model. Three types of sensitivity analysis for a linear programming model namely, Type I sensitivity (Basic invariancy), Type II sensitivity (Support set invariancy) and Type III sensitivity (optimal partition invariancy) were categorized and summarized by Koltai and Terlaky (2000) and Hadigheh and Terlaky (2006; 2007). If the optimal solution of a linear programming model is non-degenerate, all the three types are the same. Koltai and Terlaky (2000) have shown that the Type I sensitivity analysis of the degenerate linear programming model does not provide satisfactorily information. For obtaining suitable sensitivity analysis in a degenerate linear model, Type II sensitivity must be considered. Since SAP is a highly degenerate linear model, Type II sensitivity is studied in this paper.

\subsection{Cost Sensitivity Analysis}

SA of coefficients from the objective function of the SAP is a particular case of parametrical programming, where one of the cost coefficients of the objective function, $c_{i j k}$ of the decision variable $x_{i j k}$ is replaced by $c_{i j k}+\Delta_{i j k}$ and the rest of the data remain unchanged.

An SA at the costs of non basic cells do not alter the existing optimal solution, as the current cost itself is very high and there has been no allocation along this route. The SA at the costs of allocated cells is likely to change the transportation schedule. In this case, SAP is re-studied with the current optimal solution. Using the current optimal solution of SAP, the MODI-indices $u_{i}, i=1,2, \ldots, n ; v_{j}, j=1,2, \ldots, n ; w_{k}, k=1,2, \ldots, n$ can be obtained, using the basic cell property and $\delta_{i j k}=c_{i j k}-u_{i}-v_{j}-w_{k}$, for all non-basic cells are computed. Then, $c_{i j k}$ is replaced by $c_{i j k}+\Delta_{i j k}$ and the rest of the data remain unchanged and the new values for the MODI-indices $u_{i}, i=1,2, \ldots, n ; v_{j}, j=1,2, \ldots, n ; w_{k}, k=1,2, \ldots, n$ are computed. The $\mathrm{SR}$ of $\Delta_{i j k}$ is evaluated using the optimality conditions, that is, $\delta_{i j k}=c_{i j k}-u_{i}-v_{j}-w_{k} \geq 0$ for all non basic cells. The SRs of other cells can be obtained this way.

\subsection{Computation of MODI-indices}

Let $c_{i j k}+\Delta_{i j k}$ be the cost coefficient of $(\mathrm{i}, \mathrm{j}, \mathrm{k})$ th cell in the perturbed problem where $\Delta_{i j k}$ is a parameter. The optimal solution of SAP contains only $n$ basic cells, but we have $3 n$ MODI-indices.

We choose the MODI-indices $u_{i}=\theta_{i}, i=1,2,3, \ldots, n \quad$ and $v_{j}=\theta_{j}, j=n+1, n+2, \ldots, 2 n \quad$ where $\theta_{i}, i=1,2,3, \ldots, 2 n$ are parameters. By applying the conditions $c_{i j k}-\left(u_{i}+v_{j}+w_{k}\right)=0$, for all basic cells $(\mathrm{i}, \mathrm{j}$, $\mathrm{k}$ ), we can find the rest of the MODI-indices $w_{k}, k=1,2,3, \ldots, n$ as a function of $\theta_{i}{ }^{\prime} s$.

The following theorems are used in the proposed method for finding the costs SRs of basic and non-basic variables in the SAP.

Theorem 3.1: Let $(\mathrm{i}, \mathrm{j}, \mathrm{k})$ th cell be a non-basic cell corresponding to an optimal solution of the SAP with $\delta_{i j k}=c_{i j k}-u_{i}-v_{j}-w_{k}(\geq 0)$. If $c_{i j k}+\Delta_{i j k}$ is the perturbed cost of $c_{i j k}$, then the range of $\Delta_{i j k}=\left[-\delta_{i j k}, \infty\right)$.

Proof: Now, since $(\mathrm{i}, \mathrm{j}, \mathrm{k})$ th cell is a non-basic cell and the perturbed cost $c_{i j k}+\Delta_{i j k}$ is not affected the current optimal solution to the problem, $c_{i j k}+\Delta_{i j k}-u_{i}-v_{j}-w_{k} \geq 0$. This implies that, $\Delta_{i j k} \geq-\delta_{i j k}$. Therefore, the range of $\Delta_{i j k}=\left[-\delta_{i j k}, \infty\right)$ 
Hence, the theorem is proved.

Theorem 3.2: Let $(\mathrm{i}, \mathrm{j}, \mathrm{k})$ th cell be basic cell corresponding to an optimal solution of the SAP with $\delta_{i j k}=c_{i j k}-u_{i}-v_{j}-w_{k}(=0)$. If $c_{i j k}+\Delta_{i j k}$ is the perturbed value of $c_{i j k}$ and $U_{i}$ is the minimum value of $\delta_{i j k}$ for all non-basic cells in the ith origin, $V_{j}$ is the minimum value of $\delta_{i j k}$ for all non-basic cells in the jth destination and $W_{k}$ is the minimum value of $\delta_{i j k}$ for all non-basic cells in the kth conveyance, then the range of $\Delta_{i j k}=\left(-\infty, M_{i j k}\right]$ where $M_{i j k}=$ the maximum $\left\{U_{i}, V_{j}, W_{k}\right\}$.

Proof: Now, since $c_{i j k}+\Delta_{i j k}$ is the perturbed value of $c_{i j k}$ and the current optimal solution remains optimal, $\delta_{i j k}=c_{i j k}-u_{i}-v_{j}-w_{k} \geq 0$, for all non-basic cells in the ith origin, the jth destination and the kth conveyance are positive.

Now, attaching the $\Delta_{i j k}$ to first $u_{i}$, then $v_{j}$ and then $w_{k}$, we have the following:

$$
\begin{gathered}
c_{i s l}-\left(u_{i}+\Delta_{i j k}\right)-v_{s}-w l \geq 0,(\mathrm{i}, \mathrm{s}, l) \text { is non - basic cells, for all } \mathrm{s} \text { and } l \text {; } \\
c_{r j l}-u_{r}-\left(v_{j}+\Delta_{i j k}\right)-w_{l} \geq 0,(\mathrm{r}, \mathrm{j}, l) \text { is non - basic cells, for all } r \text { and } l \text { or } \\
c_{r s k}-u_{r}-v_{s}-\left(w_{k}+\Delta_{i j k}\right) \geq 0,(\mathrm{r}, \mathrm{s}, k) \text { is non - basic cells, for all } \mathrm{r} \text { and s. }
\end{gathered}
$$

Thus, we can conclude on the basis of the above implications that $\Delta_{i j k} \leq U_{i} ; \Delta_{i j k} \leq V_{j}$ or $\Delta_{i j k} \leq W_{k}$. Now, since we attach any one of the MODI-indices $u_{i}, v_{j}$ and $w_{k}$, we take, $M_{i j k}=\operatorname{maximum}\left\{U_{i}, V_{j}, W_{k}\right\}$ for getting some better range. Therefore, the range of $\Delta_{i j k}=\left(-\infty, M_{i j k}\right]$.

Hence, the theorem is proved.

\section{Parametric-Bound Technique}

We, now introduce a new procedure, namely parametric-bound technique based on the Theorem 3.1 and the Theorem 3.2 to analyze the costs SA in a SAP.

The parametric-bound technique proceeds as follows.

Step 1. Compute an optimal solution to the given SAP using the method adopted by Anuradha-Pandian (AP method) (2012).

Step 2. Assign the parameters $\theta_{i}, i=1,2, \ldots, 2 n$ to the MODI-indices such that $u_{i}=\theta_{i}, i=1,2, \ldots, n$ and $v_{j}=\theta_{j}, j=n+1, n+2, \ldots, 2 n$ and then, compute the values of the rest of MODI-indices, $w_{k}, k=1,2, \ldots, n$ as a function of $\theta_{i}$ 's.

Step 3. Construct the MODI-indices table for the optimal solution obtained in the Step1 and then, Compute $\delta_{i j k}=c_{i j k}-u_{i}-v_{j}-w_{k}$ for each non-basic cell $(\mathrm{i}, \mathrm{j}, \mathrm{k})$ which is a function of the parameters $\theta_{i}{ }^{\prime} s$.

Step 4. Compute all possible relations among the parameters $\theta_{i}, i=1,2, \ldots, 2 n$ using optimality condition, $c_{i j k}-\left(u_{i}+v_{j}+w_{k}\right) \geq 0$, for all non-basic cells $(\mathrm{i}, \mathrm{j}, \mathrm{k})$.

Step 5. Using the Theorem 3.1 and overall relations obtained in the Step 3., compute the costs range of all non-basic cells.

Step 6. Compute the costs range of all basic cells using the Theorem 3.2 and the relations obtained in the Step 4.

For easy computing and clear understanding, the proposed method will be applied directly on a table as that of classical transportation algorithm. Parameters corresponding to the cell (i, j, k) are displayed as follows: we put the cost $c_{i j k}$ at the left-side and the $\delta_{i j k}=c_{i j k}-u_{i}-v_{j}-w_{k}$ at the right-side.

The proposed technique is illustrated the following numerical example.

Example 1: Suppose that there are three men denoted by $M_{1}, M_{2}$ and $M_{3}$, three factories denoted by $F_{1}$, $F_{2}$ and $F_{3}$, and three jobs denoted by $J_{1}, J_{2}$ and $J_{3}$. It is known that $c_{i j k}$ is the assignment cost of the job $\mathrm{j}$ in the factory $\mathrm{k}$ to be performed by the man $\mathrm{i}$. Besides, three men, three factories and three jobs can be associated with only one of the others, that is, only one man is associated with only one factory with only one job. The assignment costs $c_{i j k}$ are given in the following table. 
Table 1. The assignment costs $c_{i j k}$

\begin{tabular}{cccccccccc}
\hline Jobs & & J1 & & & J2 & \multicolumn{3}{c}{ J3 } \\
\hline Mens $\downarrow /$ Factories $\rightarrow$ & F1 & F2 & F3 & F1 & F2 & F3 & F1 & F2 & F3 \\
\hline M1 & 10 & 8 & 12 & 9 & 10 & 27 & 15 & 10 & 13 \\
M2 & 8 & 6 & 7 & 9 & 6 & 12 & 7 & 11 & 12 \\
M3 & 9 & 7 & 6 & 10 & 7 & 12 & 8 & 6 & 8 \\
\hline
\end{tabular}

Now, by the AP method (2012), the optimal solution to the given solid assignment problem is $M_{3} \stackrel{F_{3}}{\longrightarrow} J_{1}$, $M_{2} \stackrel{F_{1}}{\longrightarrow} J_{3}$ and $M_{1} \stackrel{F_{2}}{\longrightarrow} J_{2}$ and the total minimum assignment cost is 23 .

Now, we take $u_{i}=\theta_{i}, i=1,2,3$ and $v_{j}=\theta_{j}, j=4,5,6$.

Now, using the basic cell condition, the values of the rest of the MODI-indices $w_{k}, k=1,2,3$ are obtained as follows: $w_{1}=7-\theta_{2}-\theta_{6} ; w_{2}=10-\theta_{1}-\theta_{5}$ and $w_{3}=6-\theta_{3}-\theta_{4}$.

Table 2. MODI-indices

\begin{tabular}{cccccccc}
\hline & & \multicolumn{2}{c}{$v_{1}=\theta_{4}$} & \multicolumn{2}{c}{$v_{2}=\theta_{5}$} & \multicolumn{2}{c}{$v_{3}=\theta_{6}$} \\
\hline \multirow{3}{*}{$u_{1}=\theta_{1}$} & $w_{1}=7-\theta_{2}-\theta_{6}$ & 10 & $\delta_{111}$ & 9 & $\delta_{12}$ & 15 & $\delta_{131}$ \\
& $w_{2}=10-\theta_{1}-\theta_{5}$ & 8 & $\delta_{112}$ & $\mathbf{1 0}$ & $\mathbf{0}$ & 10 & $\delta_{132}$ \\
& $w_{3}=6-\theta_{3}-\theta_{4}$ & 1 & $\delta_{113}$ & 27 & $\delta_{123}$ & 13 & $\delta_{133}$ \\
\hline \multirow{3}{*}{$u_{2}=\theta_{2}$} & $w_{1}=7-\theta_{2}-\theta_{6}$ & 8 & $\delta_{211}$ & 9 & $\delta_{221}$ & $\mathbf{7}$ & $\mathbf{0}$ \\
& $w_{2}=10-\theta_{1}-\theta_{5}$ & 6 & $\delta_{212}$ & 6 & $\delta_{222}$ & 11 & $\delta_{232}$ \\
$u_{3}=\theta_{3}$ & $w_{3}=6-\theta_{3}-\theta_{4}$ & 7 & $\delta_{213}$ & 12 & $\delta_{223}$ & 12 & $\delta_{233}$ \\
\hline & $w_{2}=7-\theta_{2}-\theta_{6}$ & 9 & $\delta_{311}$ & 10 & $\delta_{321}$ & 8 & $\delta_{331}$ \\
& $w_{3}=6-\theta_{3}-\theta_{4}$ & $\mathbf{6}$ & $\delta_{312}$ & 7 & $\delta_{322}$ & 6 & $\delta_{332}$ \\
\hline
\end{tabular}

where $\delta_{111}=3-\theta_{1}+\theta_{2}-\theta_{4}+\theta_{6} ; \quad \delta_{112}=-2-\theta_{4}+\theta_{5} ; \quad \delta_{113}=6-\theta_{1}+\theta_{3} ; \quad \delta_{121}=2-\theta_{1}+\theta_{2}-\theta_{5}+\theta_{6} \quad$; $\delta_{123}=21-\theta_{1}+\theta_{3}+\theta_{4}-\theta_{5} ; \quad \delta_{131}=8-\theta_{1}+\theta_{2} ; \quad \delta_{132}=\theta_{5}-\theta_{6} ; \quad \delta_{133}=7-\theta_{1}+\theta_{3}+\theta_{4}-\theta_{6} ;$

$\delta_{211}=1-\theta_{4}+\theta_{6} ; \quad \delta_{212}=-4+\theta_{1}-\theta_{2}-\theta_{4}+\theta_{5} ; \quad \delta_{213}=1-\theta_{2}+\theta_{3} ; \quad \delta_{221}=2-\theta_{5}+\theta_{6} ; \delta_{222}=-4+\theta_{1}-\theta_{2} ;$ $\delta_{223}=6-\theta_{2}+\theta_{3}+\theta_{4}-\theta_{5} ; \quad \delta_{232}=1+\theta_{1}-\theta_{2}+\theta_{5}-\theta_{6} ; \delta_{233}=6-\theta_{2}+\theta_{3}+\theta_{4}-\theta_{6} ;$

$\delta_{311}=2+\theta_{2}-\theta_{3}-\theta_{4}+\theta_{6} ; \quad \delta_{312}=-3+\theta_{1}-\theta_{3}-\theta_{4}+\theta_{5} ; \quad \delta_{321}=3+\theta_{2}-\theta_{3}-\theta_{5}+\theta_{6} \quad ; \quad \delta_{322}=-3+\theta_{1}-\theta_{3} \quad ;$ $\delta_{323}=6+\theta_{4}-\theta_{5} ; \quad \delta_{331}=1+\theta_{2}-\theta_{3} ; \quad \delta_{332}=-4+\theta_{1}-\theta_{3}+\theta_{5}-\theta_{6}$ and $\delta_{333}=2+\theta_{4}-\theta_{6}$.

Now, by the optimality conditions, that is, $c_{i j k}-u_{i}-v_{j}-w_{k} \geq 0$ for all non-basic cells (i, $\mathrm{j}, \mathrm{k}$ ), we obtain the following overall results:

$$
\begin{array}{ccc}
-1 \leq \theta_{1}-\theta_{2}+\theta_{5}-\theta_{6} \leq 2 ; & 4 \leq \theta_{1}-\theta_{2} \leq 8 ; & -6 \leq \theta_{4}-\theta_{5} \leq-2 ; \\
0 \leq \theta_{5}-\theta_{6} \leq 2 ; & 3 \leq \theta_{1}-\theta_{3} \leq 6 ; & 3 \leq \theta_{1}-\theta_{3}-\theta_{4}+\theta_{5} \leq 6 ; \\
-2 \leq \theta_{4}-\theta_{6} \leq 1 ; & -1 \leq \theta_{2}-\theta_{3} \leq 1 ; & -2 \leq \theta_{2}-\theta_{3}-\theta_{4}+\theta_{6} \leq 6 .
\end{array}
$$

Now, using the Theorem 3.1 and the Theorem 3.2 and also, the above relations, we obtain the following the Type II ranges of $4_{i j k}{ }^{\prime} s$ in the given SAP. 
Table 3. Type II ranges of $4_{j k}{ }^{\prime} s$

\begin{tabular}{ccccc}
\hline & & $J_{1}$ & $J_{2}$ & $J_{3}$ \\
\hline \multirow{3}{*}{$M_{1}$} & $F_{1}$ & {$[-1, \infty)$} & {$[-3, \infty)$} & {$[-4, \infty)$} \\
& $F_{2}$ & {$[-4, \infty)$} & $(-\infty, 2]$ & {$[-2, \infty)$} \\
& $F_{3}$ & {$[-3, \infty)$} & {$[-18, \infty)$} & {$[-5, \infty)$} \\
\multirow{3}{*}{$M_{2}$} & $F_{1}$ & {$[-3, \infty)$} & {$[-2, \infty)$} & $(-\infty, 2]$ \\
& $F_{2}$ & {$[-10, \infty)$} & {$[-4, \infty)$} & {$[-3, \infty)$} \\
& $F_{3}$ & {$[-2, \infty)$} & {$[-5, \infty)$} & {$[-8, \infty)$} \\
$M_{3}$ & $F_{1}$ & {$[-8, \infty)$} & {$[-4, \infty)$} & {$[-2, \infty)$} \\
& $F_{2}$ & {$[-18, \infty)$} & {$[-3, \infty)$} & {$[-4, \infty)$} \\
& $F_{3}$ & $(-\infty, 2]$ & {$[-4, \infty)$} & {$[-3, \infty)$} \\
\hline
\end{tabular}

\section{Acknowledgements}

The authors thank to Dr. G. Natarajan and Prof. M. A. Mohamed Sahul Hameed, VIT University, Vellore for their valuable comments and suggestions, which were very helpful in improving the presentation of this paper.

\section{References}

Anuradha, D., \& Pandian, P. (2012). A new method for finding an optimal solution to solid assignment problems. International Journal of Engineering Research and Applications, 2, 1614-1618.

Frieze, A. M., \& Yadegar, L. (1981). An algorithm for solving 3-dimensional assignment problems with application to scheduling in a teaching practice. Journal Oper. Res. Soc., 32, 989-995.

Hadigheh, A. G., \& Terlaky, T. (2006). Sensitivity analysis in linear optimization: invariant Support set intervals. European Journal of Operational Research, 169, 1158-1175. http://dx.doi.org/10.1016/j.ejor.2004.09.058

Hadigheh, A. G., \& Terlaky, T. (2007). Active constraint set invariancy sensitivity analysis in linear optimization. Journal of Optimization Theory and Applications, 133, 303-315. http://dx.doi.org/10.1007/s10957-007-9201-5

Koltai, T., \& Terlaky, T. (2000). The Difference between the Managerial and Mathematical Interpretation of Sensitivity Analysis Results in Linear Programming. International Journal of Production Economics, 65, 257-274. http://dx.doi.org/10.1016/S0925-5273(99)00036-5

Kuhn, H. W. (1955). The Hungarian method for the assignment and transportation problem. Naval Research Logistics Quarterly, 2, 83-97. http://dx.doi.org/10.1002/nav.3800020109

Lin, C. J., \& Wen, U. P. (2003). Sensitivity analysis of the optimal assignment problem. European Journal of Operational Research, 149, 35-46. http://dx.doi.org/10.1016/S0377-2217(02)00439-3

Lin, C. J., \& Wen, U. P. (2007). Sensitivity analysis of objective function coefficients of the assignment problem. Asia-Pacific Journal of Operational Research, 24, 203-221. http://dx.doi.org/10.1142/S0217595907001115

Lin, C. J., Wen, U. P., \& Lin, P. Y. (2011). Advanced sensitivity analysis of the fuzzy assignment problem. Applied Soft Computing, 11, 5341-5349. http://dx.doi.org/10.1016/j.asoc.2011.05.025

Pierskalla, W. P. (1968). The multidimensional assignment problem. Oper. Research, 16, 422-431. http://dx.doi.org/10.1287/opre.16.2.422

Votaw, D. F., \& Orden, A. (1952). The personal assignment problem. Symposium on linear inequalities and programming, 155-163. 